\title{
Upper Extremity Deep Vein Thrombosis After Botulinum Toxin Injection: A Case Report
}

\author{
Nana Lim, MD, Geun Su Lee, MD, Ki Hong Won, MD, Jin Sun Kang, MD, Sung Hoon Lee, MD, \\ Eun Young Kang, MD, Hyun Kyung Lee, MD, PhD, Youn Kyung Cho, MD \\ Department of Rehabilitation Medicine, Kwangju Christian Hospital, Gwangju, Korea
}

\begin{abstract}
Botulinum toxin (BoNT) injection is widely used to improve spasticity. However, after the treatment, the patient may experience pain, inflammation, swelling and redness at the injection site. In this case, we addressed deep vein thrombosis (DVT) after BoNT treatment of the upper limb. A male aged 37 years had spasticity and dystonia in his left upper extremity. BoNT-A $100 \mathrm{U}$ was injected into the left biceps brachii and an equal amount into the brachialis to relieve spasticity. After three days, he developed redness and painful swelling in the left upper arm and the next day, through the upper extremity computed tomography venography, DVT was identified in the left cephalic vein. The thrombus resolved after the anticoagulation therapy with rivaroxaban (Xarelto). We hypothesized the role of mainly three mechanisms in the development of DVT in this case: repetitive strenuous activity, relative stasis due to reduced muscle tone, and possible direct mechanical damage to the vessel wall.
\end{abstract}

Keywords Upper extremity DVT, Botulinum toxins, Muscle spasticity

\section{INTRODUCTION}

Spasticity after central nervous system injury is a common complication, with a reported occurrence of $4 \%$ to $42.6 \%$ in stroke patients. It could lead to various impairments in the reduction of the daily life functions, pain, secondary skin disease, and so on. It increases the burden of patient care, such as the difficulty in changing the position of his or her body or handling the feces and urine. There are various methods for treating spasticity, such as passive stretching, pharmacologic management, chemodenervation, intrathecal therapies, and the surgical intervention. Among them, botulinum toxin injection may temporarily reduce spasticity, particularly in patients with focal spasticity. Furthermore, injection is not as invasive as surgical treatment. It has a better therapeutic effect with fewer side effects on the target area than oral medications, having systemic consequences. Therefore, it is a treatment of choice causing marked improvement in spasticity if proper technique is followed.

Received June 8, 2020; Revised August 28, 2020; Accepted October 6, 2020; Published online April 14, 2021

Corresponding author: Youn Kyung Cho

Department of Rehabilitation Medicine, Kwangju Christian Hospital, Yangrimro 37, Namgu, Gwangju 61661, Korea. Tel: +82-62-650-5167, Fax: +82-62671-7447, E-mail: 10191@kchgw.org

ORCID: Nana Lim (https://orcid.org/0000-0001-5743-9135); Geun Su Lee (https://orcid.org/0000-0003-2131-3148); Ki Hong Won (https://orcid. org/0000-0002-1456-7943); Jin Sun Kang (https://orcid.org/0000-0003-0507-4044); Sung Hoon Lee (https://orcid.org/0000-0002-2071-7936); Eun Young Kang (https://orcid.org/0000-0001-6805-5727); Hyun Kyung Lee (https://orcid.org/0000-0002-1464-2560); Youn Kyung Cho (https://orcid. org/0000-0003-4906-6089).

(c) This is an open-access article distributed under the terms of the Creative Commons Attribution Non-Commercial License (http://creativecommons.org/ licenses/by-nc/4.0) which permits unrestricted noncommercial use, distribution, and reproduction in any medium, provided the original work is properly cited. Copyright ( 2021 by Korean Academy of Rehabilitation Medicine 
Botulinum toxin is widely used in spasticity due to the pharmacological action of lowering muscle tone by interfering with the secretion of acetylcholine from the presynaptic terminal plate acting on the neuromuscular junction. Therefore, a number of researches have been conducted about its treatment methods and treatment variables. Typical side effects after botulinum toxin injection include dysphagia, muscle weakness, allergic reactions, flu-like symptoms, and injection site trauma [1]. Thromboembolic events are rarely described as reported adverse reactions [1-3]. Cote et al. [1] reviewed all (therapeutic and cosmetic use) serious adverse events to the US Food and Drug Administration (FDA) from December 1989 to May 2003, and only two cases of pulmonary embolism were reported. Recently, we experienced a case of deep vein thrombosis (DVT) of the upper extremity, diagnosed 4 days after botulinum toxin injection. We describe a case of upper extremity DVT after botulinum toxin injection with a discussion of etiology, diagnosis, and management. To our knowledge, this is the first report of the upper extremity DVT after botulinum toxin type A (BoNT-A) injection. This case report was approved by the Institutional Review of Board of Kwangju Christian Hospital (No. KCH-RE-2019-09-003), which waived the requirement for imformed consent owing to the retrospective nature of this study.

\section{CASE REPORT}

A male patient aged 37 years visited the local hospital and planned to undergo botulinum toxin injection to relieve spasticity and dystonia of his left upper limb 3 years after subarachnoid hemorrhage. His past medical history

Table 1. Patient's coagulation profile

\begin{tabular}{lc}
\hline \multicolumn{1}{c}{ Parameter } & \multicolumn{1}{c}{ Value } \\
\hline Prothrombin time (s) & $12.9(9.8-12.7)$ \\
Prothrombin time (INR) & $1.12(0.85-1.15)$ \\
Prothrombin time (\%) & $71.2(75-130)$ \\
Activated partial thromboplastin time (s) & $30.1(23.0-39.0)$ \\
D-dimer $(\mu \mathrm{g} / \mathrm{mL})$ & $2.2(0-1.0)$ \\
Fibrin degradation products $(\mu \mathrm{g} / \mathrm{mL})$ & $2.7(0-5)$ \\
Antithrombin III $(\%)$ & $114.6(75-125)$ \\
Bleeding time $(\mathrm{min})$ & $3(1-5)$ \\
\hline
\end{tabular}

Values are presented as median (range).

INR, international normalized ratio. revealed the fact that he had been affected by dystonia and spasticity in his left upper limb, especially the hand, secondary to tuberculous meningitis at the age of 3 . The patient reported that for decades his left upper limb muscle strength was normal, however, it decreased to fair grade after the subarachnoid hemorrhage with worsening of dystonic and spastic symptom.

The degree of spasticity before the treatment was Modified Ashworth Scale (MAS) 2 in upper arm flexor muscles. The patient received a total of $200 \mathrm{U}$ of onabotulinum toxin A (Botox): $100 \mathrm{U}$ each into the left biceps brachii muscle and the brachialis muscle to relieve spasticity. Guiding techniques such as the ultrasound or electromyography were not used, but no complications were noted during injection. Electrical stimulation therapy was applied to the treated muscle, and the patient stretched his arm repeatedly to improve the range of motion. He performed exercises of the arm and shoulders at least 3 hours daily at a higher intensity than usual. During the exercise, there was no discomfort, and later, he increased exercise intensity. However, 3 days later, progressive edema and pain occurred in the left upper limb and he was transferred to our hospital the next day. He had no history of trauma except the stretching exercise, with no history of previous cardiovascular or other hemorrhagic diseases. He did not take drugs that affecting blood coagulation tendency. There were no known drug allergies. The thrombophilia profile was negative except for

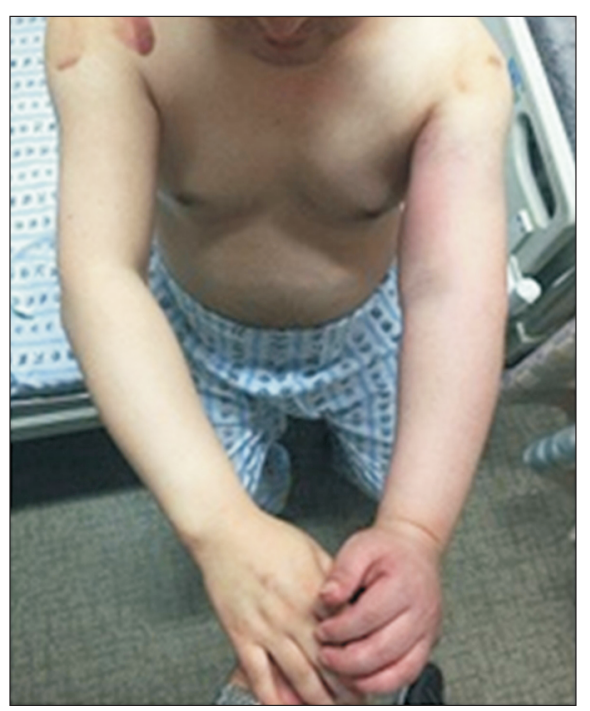

Fig. 1. Erythematous swelling on the left hand, forearm, and shoulder region. 
the mildly elevated D-dimer concentration $(2.2 \mu \mathrm{g} / \mathrm{mL})$ (Table 1).

The strength of the left shoulder flexor and extensor muscle at the time of visiting our clinic was fair grade (manual muscle test, 3/5). The spasticity of the upper arm flexor muscle was identified as MAS 1+. Physical examination revealed edema, heat and tenderness of the left upper extremity (Fig. 1). No sign of a local infection or tenderness was observed in the area of BoNT-A injection conducted 4 days back. The circumferences measured bilaterally at $5 \mathrm{~cm}$ above medial epicondyle were $33.5 \mathrm{~cm}$ on right and $37.6 \mathrm{~cm}$ on left, respectively. Given the patient's symptoms, DVT was suspected, and thus, Doppler ultrasonography was performed. The results revealed the presence of DVT at the lower region of the left brachial and axillary veins (Fig. 2). In addition, venous computed tomography (CT) angiography was conducted to identify the distribution of thrombosis. Thus, the thrombus was observed from the lower region of internal jugular vein including the brachiocephalic and the axillary veins (Fig. 3).

The patient was placed in a sling to immobilize the arm and anticoagulation therapy was initiated with rivaroxaban (Xarelto; $15 \mathrm{mg}$ twice daily per oral). After 1 week, compressive therapy was combined using compression bandage with icepack to reduce febrile sensation and edema. The edema in the upper extremity improved with time and the pain with febrile sensation disappeared.

At the 2-week follow-up, the patient had no pain or swelling. CT performed one month later revealed the resolution of filling defect with only small residual thrombosis in the brachiocephalic vein (Fig. 4). To reduce the risk of recurrence of DVT, rivaroxaban (Xarelto) was continued at the dosage of $20 \mathrm{mg}$ once daily for 6 months.

\section{DISCUSSION}

Virchow's triad is a well-known theory which explaining
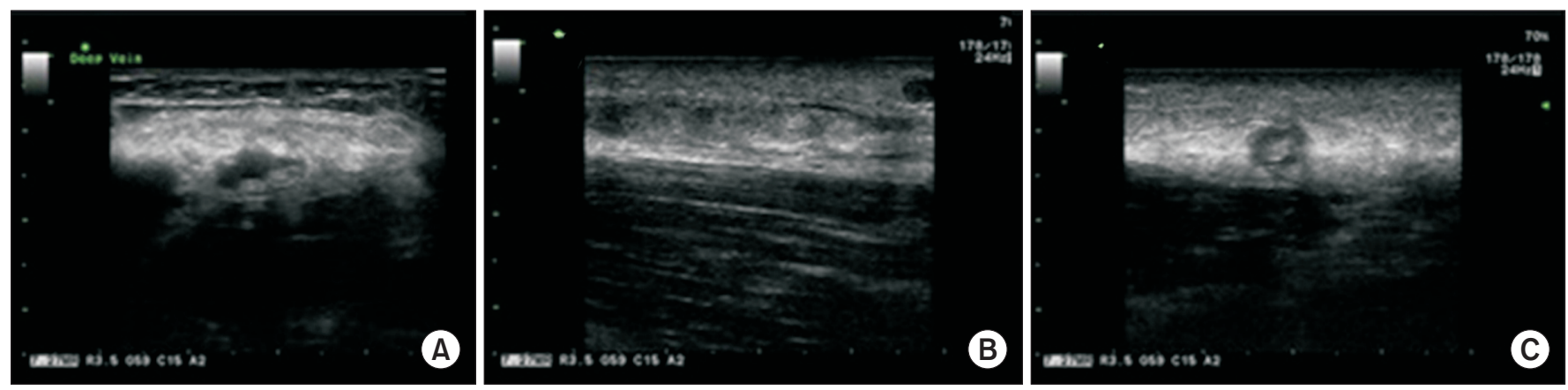

Fig. 2. Ultrasound showed deep vein thrombosis in the brachial vein (A), axillary vein (B), and superficial cephalic vein $(\mathrm{C})$.
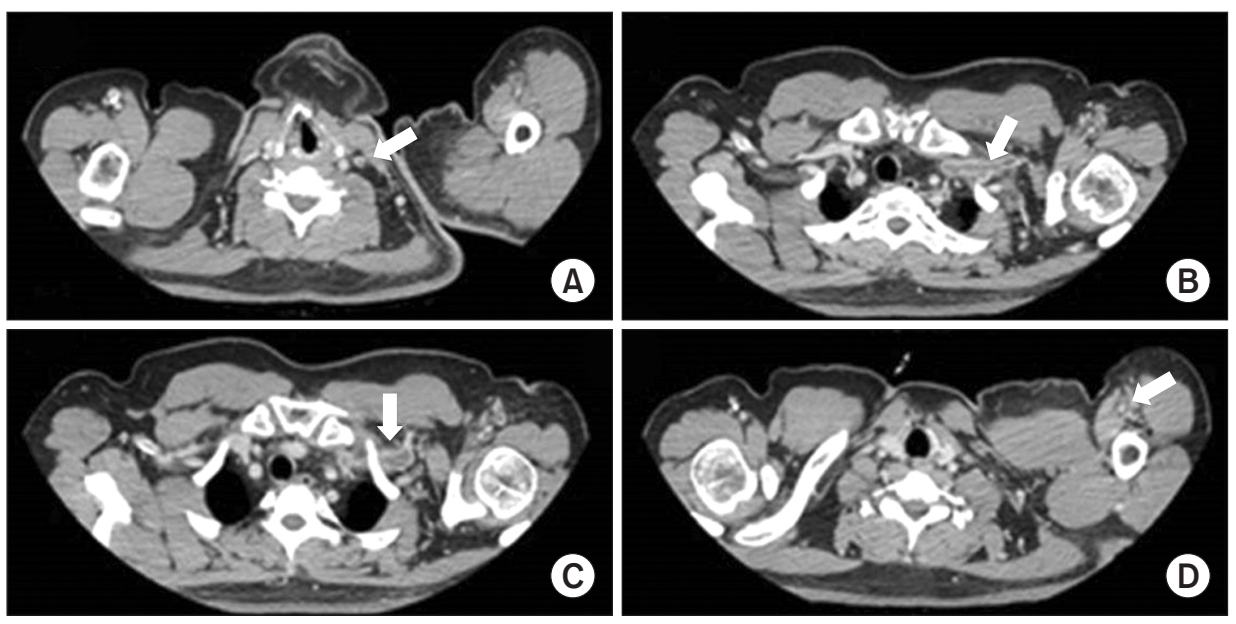

Fig. 3. Computed tomography venography showed deep vein thrombosis in the internal jugular vein $(A)$, brachiocephalic vein (B), axillary vein (C), and brachial vein (D) in the left extremity of the patient. 

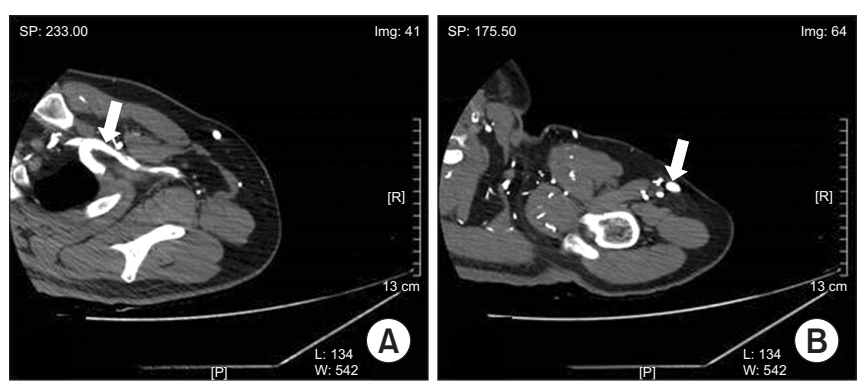

Fig. 4. Computed tomography venography performed 1 month later revealed a resolution of filling defect with only small residual thrombosis in the brachiocephalic vein (A) and brachial vein (B).

venous thrombus formation and is primarily composed of three factors: blood flow congestion, blood coagulation, and vascular wall damage. The DVT of the upper limbs accounts for approximately $4 \%$ to $10 \%$ of the total DVTs. As DVT in the upper extremity is relatively rare, it is less studied than in the lower extremities. Recently, more attempts have been made to probe the pathogenesis and clinical meaning of the upper extremity DVT. Kucher [4] categorized upper extremity DVT into two subgroups on the basis of pathogenesis: a primary and a secondary form due to obvious underlying causes, mainly cancer and indwelling central venous catheters. He spilt the primary upper extremity into three subtypes of venous thoracic outlet syndrome, effort-related thrombosis, and idiopathic type which has no relation to the thoracic outlet syndrome or to exertion. The author suggested mechanisms of venous thoracic outlet syndrome as compression of the subclavian vein resulting from abnormality of structures. Effort-related thrombosis, accounting for approximately two-thirds of the primary upper extremity, is defined as the direct damage to the vein from muscular stretching, later termed as "Paget-Schroetter syndrome" $[4,5]$.

In this case report, although the cause of DVT in the patient was unclear, the probable rationales were as follows.

First, the patient did not have the mentioned anatomical abnormalities, mechanisms of venous thoracic outlet syndrome such as compression by the first rib, clavicle, subclavius muscle, costoclavicular ligament, or anterior scalene muscle. However, he underwent repetitive and highly intensive passive range of motion exercise from the day after the botulinum toxin injection. As the spasticity progressively improved, his exercise intensity and duration subsequently increased. During exercise, the vessels with a constant length for many years, stretched extensively, possibly resulting in intimal injury in blood vessels. Recurrent microtrauma in the subclavian vein, as in effortful DVT, might have affected the etiology and triggered fibrosis and extrinsic constriction of the vessel wall. Generally, the exercise-induced venous thrombosis is observed in athletes involved in a wide variety of sports such as ball games, combatant sport and heavy athletics, games with rackets or clubs, and aquatic sports [6].

Second, the tone reduction after the injection might have contributed to the relative venous stasis. Spasticity improves venous return, thereby minimizing the development of thrombophlebitis, similar to that observed in patients with spinal cord injury. In these patients, spasticity conveys some protection against DVT [7].

Lastly, there might be direct vascular injury during the injection procedure. Even though there were no complications soon after the procedure, there remains the possibility of needle-induced mechanical damage because guide technique such as ultrasound was not used during the procedure $[2,3]$.

Recently, there were two case reports of thrombosis after botulinum toxin injection. First, Mines et al. [2] reported a case of the lower extremity DVT after BoNT-A for spasticity. The patient's characteristics were similar as she was spastic and dystonic secondary to cerebral palsy caused by neonatal anoxia. In this case, the author suggested a direct effect on vascular smooth muscles and relief of muscle hypertonia as possible explanations for DVT formation. However, the additional suggestion given by the author of decreased mobility playing a role is contrary to our case. The author accessed the interval between the BoNT-A injection and the onset of lower extremity DVT as "plausible" according to the World Health Organization-Uppsala Monitoring Centre (WHO-UMC) causality assessment system [8]. In our case, the formation of DVT is not an objective and specific medical disorder or a recognized pharmacological phenomenon. Although the interval between the start of the drug and the onset of the event is "plausible," this case report belongs to "probable" according to the WHO-UMC causality assessment system $[8,9]$.

Second, Pisani et al. [3] described a case of the axillary vein thrombosis after BoNT-A for hyperhidrosis in a female patient aged 32 years. Similar to the previous case 
report, the author suggested causality as reduced tissue tone surrounding the axillary vein, post-multiple injection traumatic inflammation, and stasis. However, in that case report, the meaning of "stasis" was unclear. Moreover, the patient had no underlying disease, which was different from the previous one and ours.

With respect to the effects of botulinum toxin on the vessel wall itself, several laboratory studies of flap viability in animal models [10] reported botulinum toxin has vasodilatory effect and preventing vasospasm and thrombosis. The mechanism of the effect is that it influences the neurons of the autonomic nervous system, resulting in increase of vessel diameter and blood perfusion.

However, in clinical setting, we assumed that the vasodilatory effect of botulinum toxin when injected into spastic muscles, not flaps, may impede the velocity of venous return. Because spasticity is known to improve venous return, reducing the incidence of thrombosis [7]. Therefore, establishing the clinical evidence whether BoNT increase or decrease the formation of DVT would be a challenge depending on the situation. Additional work is required to confirm and explain the effect of BoNT about formation of DVT.

In conclusion, there are a variety of treatments to control post-stroke spasticity, and among them, the dramatic changes in spasticity may occur after neural interventions such as the BoNT-A injection. To our knowledge, this is the first case report of the upper extremity DVT after BoNT-A injection. Although generally safe, clinicians should be careful during injection. An ultrasonography guide is recommended to avoid potential mechanical vessel wall injury and localize the target muscles. In particular, patients should be recommended progressive range of motion exercise due to possible excessive stretch on vessel intima layer.

\section{CONFLICT OF INTEREST}

No potential conflict of interest relevant to this article was reported.

\section{AUTHOR CONTRIBUTION}

Conceptualization: Cho YK, Lim NN. Methodology: Cho YK, Kang EY, Lee GS. Formal analysis: Lee HK, Won KH, Lee GS. Project administration: Cho YK, Lim NN,
Kang JS. Visualization: Cho YK, Lim NN, Won KH. Writing - original draft: Cho YK, Lim NN, Kang JS, Lee GS. Writing - review and editing: Cho YK, Lim NN, Lee SH. Approval of the final manuscript: all authors.

\section{REFERENCES}

1. Cote TR, Mohan AK, Polder JA, Walton MK, Braun MM. Botulinum toxin type A injections: adverse events reported to the US Food and Drug Administration in therapeutic and cosmetic cases. J Am Acad Dermatol 2005;53:407-15.

2. Mines ML, Pacheco T, Castel-Lacana E, de Boissezon X, Marque P, Montastruc F. Venous thrombosis after botulinum therapy in lower limb: a case report and literature review. Ann Phys Rehabil Med 2019;62:457-8.

3. Pisani LR, Bramanti P, Calabro RS. A case of thrombosis of subcutaneous anterior chest veins (Mondor's disease) as an unusual complication of botulinum type A injection. Blood Coagul Fibrinolysis 2015;26:685-6.

4. Kucher N. Clinical practice: deep-vein thrombosis of the upper extremities. N Engl J Med 2011;364:861-9.

5. Illig KA, Doyle AJ. A comprehensive review of PagetSchroetter syndrome. J Vasc Surg 2010;51:1538-47.

6. Zell L, Kindermann W, Marschall F, Scheffler P, Gross J, Buchter A. Paget-Schroetter syndrome in sports activities: case study and literature review. Angiology 2001;52:337-42.

7. Green D, Hartwig D, Chen D, Soltysik RC, Yarnold PR. Spinal Cord Injury Risk Assessment for Thromboembolism (SPIRATE Study). Am J Phys Med Rehabil 2003;82:950-6.

8. Uppsala Monitoring Centre. The use of the WHOUMC system for standardized case causality assessment [Internet]. Geneva, Switzerland: World Health Organization; 2013 [cited 2021 Mar 1]. Available from: https://www.who.int/publications/m/item/WHOcausality-assessment.

9. Moore N, Berdai D, Blin P, Droz C. Pharmacovigilance: the next chapter. Therapie 2019;74:557-67.

10. Fathi M, Fathi H, Mazloumi M, Khalilzadeh O, Amanpour S, Meysamie A, et al. Preventive effect of botulinum toxin A in microanastomotic thrombosis: a rabbit model. J Plast Reconstr Aesthet Surg 2010;63:e720-4. 